\title{
Article \\ Linking Changes in Contraceptive Use to Declines in Teen Pregnancy Rates
}

\author{
Jennifer Manlove ${ }^{1, *}$, Quentin Karpilow ${ }^{1}$, Kate Welti ${ }^{1}$ and Adam Thomas ${ }^{1,2}$ \\ Received: 5 October 2015; Accepted: 21 December 2015; Published: 24 December 2015 \\ Academic Editor: Naomi Farber \\ 1 Reproductive Health \& Family Formation, Child Trends, Bethesda, MD 20814, USA; \\ qkarpilow@childtrends.org (Q.K.); kwelti@childtrends.org (K.W.); Adam.Thomas@georgetown.edu (A.T.) \\ 2 McCourt School of Public Policy, Georgetown University, Washington, DC 20057, USA \\ * Correspondence: jmanlove@childtrends.org; Tel.: +1-240-223-9262
}

\begin{abstract}
Using a unique microsimulation tool, Teen FamilyScape, the present study explores how changes in the mix of contraceptive methods used by teens contributed to the decline in the U.S. teen pregnancy rate between 2002 and 2010. Results indicate that changes in contraceptive use contributed to approximately half of the decline in the teen pregnancy rate during this time period $(48 \%)$ and that a little more than half of this "contraceptive effect" was due to an increase in teen condom use (58\%). The remaining share of the contraceptive effect can be attributed to an increase in the use of more effective hormonal (pill, patch, ring) and long-acting reversible contraceptive (LARC)/injectable methods (Intrauterine Devices (IUD), implant and injectable). Results from an additional counterfactual analysis suggest that the contraceptive effect was driven by the fact that the percentage of teens using no birth control fell during the study time period, rather than by the fact that some teens switched from less effective methods (condoms) to more effective hormonal and LARC/injectable methods. However, very high typical use failure rates for teen condom users suggest the need for a two-pronged approach for continuing reductions in teen pregnancy for sexually active teens: first, targeting the youth most at risk of not using contraception and helping them choose contraception, and second, increasing the effectiveness of method use among existing contraceptors.
\end{abstract}

Keywords: teen pregnancy; contraceptive methods; historical trends

\section{Introduction}

The teen pregnancy rate in the U.S. has declined dramatically over the last two decades [1], declining by almost one-third (31\%) between 2000 and 2010 (the most recent year for which data are available) from 83.4 pregnancies per 1000 teenaged women to 57.4 pregnancies per 1000 teenaged women. This decline is the result of two underlying trends: reductions in the percentage of teenagers who are sexually active and improvements in contraceptive use among teens who are sexually active. There has been some debate about whether recent declines in teen pregnancies are due primarily to increases in abstinence or to improvements in contraceptive use. A number of studies have attempted to parse out the relative importance of these trends. These studies generally find that both factors have contributed to the reduction in teen pregnancies. The estimated magnitudes of the abstinence and contraceptive effects, however, differ, based on the specific time period studied, the dataset used, and the way in which sexual activity and contraceptive use are measured. For example, two studies that focused primarily on trends in the 1990s found that declines in sexual activity had the greatest impact on reductions in teen pregnancy. Mohn et al., [2] found that the decline in teens engaging in sex accounted for $67 \%$ of the drop. Meanwhile, Santelli et al., [3] calculated that $53 \%$ of the decline in 
teen pregnancy rates among high school students could be attributed to a higher percentage delaying sexual initiation and the rest could be attributed to improved contraceptive use.

However, more recent studies, using data into the 2000s, found that trends in contraceptive use had a greater impact on reductions in teen pregnancies or births. Santelli et al., [4] found that improvements in contraceptive use accounted for $86 \%$ of the reduction in teen pregnancy between 1995 and 2002 for teens aged 15-19. The authors also found that improved contraceptive use accounted for $77 \%$ of the reduction in pregnancies among teens aged 15-17. Kearney and Levine [5] studied the related drop in teen births between 1991 and 2007 among high school students and found that increased contraceptive use was responsible for $65 \%$ of the decline. Additionally, a review by the Guttmacher Institute found there was no decline in the share of teens who were sexually experienced between 2003 and 2010, but found evidence of improvements in contraceptive use (reductions in non-use combined with increases in the use of more effective methods). The report concluded that the decline in the teen pregnancy rate during that time period was due primarily to improvements in contraceptive use [6].

Although estimates differ based on time period and measurement, all of these studies found that a substantial percentage of declines in teen pregnancies or births were due to changes in contraceptive use among teens. However, none of the papers described above examined which changes in contraceptive use contributed most to the overall contraceptive effect. This is an important gap in the literature because some changes in contraceptive use have greater impacts than others. For example, long-acting reversible contraceptive (LARC) methods, such as Intrauterine Devices (IUDs) and subdermal implants are notably more effective than other, more user-dependent methods such as condoms and oral contraception. Because they are less susceptible to user error, these methods' perfect-use failure rates (the annual rates of pregnancy among women who use their methods consistently and correctly at each act of intercourse) and their typical-use failure rates (the average annual pregnancy rates accounting for the fact that many women do not always use their method correctly and consistently) are both less than 1\% [7]. On the other hand, among pill users, the perfect-use failure rate is about the same as for long-acting methods, but the typical-use failure rate is higher, at $9 \%$ [7]. Among condom users, the perfect-use failure rate is only $2 \%$, while the typical-use failure rate is $18 \%$ [7]. Studies have found that teens are less likely than adults to be perfect users of their chosen birth control method and therefore experience even higher failure rates when relying on user-dependent methods [8-10]. Therefore, increasing the percentage of teen women using highly effectively LARCs can further reduce pregnancies versus increasing the percentage that use condoms.

While there is considerable variation in different methods' failure rates, even the least effective methods can substantially reduce the risk of pregnancy, relative to the use of no method. Our analyses of the 2011-2013 wave of the National Survey of Family Growth (NSFG) suggest that $12 \%$ of teens who were sexually active in the past three months did not use contraception at last sex. The annual rate of pregnancy is estimated to be $85 \%$ among sexually active women who do not use contraception [7]. It might be possible, then, to achieve meaningful further reductions in teen pregnancy without large increases in the use of the most effective (but the least prevalent) forms of contraception. For example, previous research has found that dramatic reductions in the pregnancy rate can be achieved by increasing the use of condoms among those who are using no method of contraception [11]. We shed light on this issue by documenting the changes in teens' use of long-acting methods and various other forms of contraception that accompanied the precipitous drop in teen pregnancies over the last decade. We then use a unique microsimulation model, Teen FamilyScape, to estimate the way in which changes in teens' contraceptive behaviors contribute to population-wide changes in pregnancy rates. The results of these analyses allow us to assess which of these changes were the most important to the reduction in the teenage pregnancy rate between 2002 and 2010. We chose this time period in part because of recent changes in contraceptive method use 
among teens. Additionally, our focus on these years allows us to study the more recent decline in teen pregnancy, thereby expanding on previous work that focused on the 1990s and early 2000s.

\section{Methods}

Teen FamilyScape was developed by researchers at Child Trends, Georgetown University, and The Brookings Institution ${ }^{1}$. The model is designed to reproduce real-world fertility-related behaviors and outcomes among teenagers in the United States. We use data from a range of sources $^{2}$ to ensure that we realistically simulate the rate at which teenage women have sex; the frequency with which sexually active teens use contraception; the types of male-controlled and female-controlled contraception that they use; the number of teens who switch onto and off of various contraceptive methods; the frequency with which teens using various types of contraception (or none at all) become pregnant; the share of teen pregnancies that result in live births, abortions, and fetal losses; and the gestation and postpartum infertility periods for each of these pregnancy outcomes.

Figure 1 diagrams Teen FamilyScape's three simulation stages. The model has a daily periodicity, which is to say that each increment in analysis time corresponds to a single day. In the first stage of the simulation, we use the female respondent file of the 2006-2010 National Survey of Family Growth $(\mathrm{NSFG})^{3}$ - a nationally representative survey that contains extensive information on sexual activity, contraceptive use, and fertility outcomes - to populate the model with a group of teenage women whose demographic characteristics are nationally representative with respect to marital status, age, race, educational attainment, and socioeconomic status [12]. Most simulated behaviors and outcomes vary according to these demographic attributes.

In the second simulation stage, we use data from the NSFG to model sexual and contraceptive behavior. Teen FamilyScape realistically models distributions of the number of months in which teen women are sexually active and the number of days in which they have intercourse during sexually active months. The model also produces realistic distributions of teenage contraceptive use. We simulate the use of three different categories of female-controlled contraception: LARC/injectable methods, including IUDs, implants, and injectables; female sterilization ${ }^{4}$; and other female-controlled methods, such as the pill, contraceptive patch, or vaginal ring (PPR $)^{5}$. With respect to male-controlled methods, we simulate the use of condoms, withdrawal and male sterilization ${ }^{6}$. We would have preferred to place injectables in their own category, as they have higher failure rates than IUDs and implants. However, sample size restrictions prevented us from doing so. We do, however, account for differential changes over time in the use of injectables versus IUDs and implants (explained in the next section). The model allows teens to switch contraceptive methods over the course of the simulation.

1 Teen FamilyScape is an extension of FamilyScape 3.0, a model of pregnancy and childbearing that was developed by the same group of researchers. While FamilyScape 3.0 focuses on all women aged 15-44, Teen FamilyScape focuses only on the teenage population and is therefore better equipped to simulate teen-specific fertility dynamics. For more information on FamilyScape 3.0, see Thomas and Karpilow (2015).

2 As described, authors' analyses of the NSFG were used to develop many of the model's parameters. More information on the NSFG and the relevant sexual activity and contraceptive measures can be found in the Appendix A. The remaining parameters are based on published data. Sources are outlined, and cited, in the Methods section.

3 The most recently available NSFG data are from the cycle that began in 2011. We did not use data from this more recent cycle to develop Teen FamilyScape because published teen pregnancy rate estimates for this period are not yet available. Thus, we currently lack the external benchmarks that would be needed to validate a model parameterized using data from the 2011-2013 NSFG cycle.

4 We consider teen women to be sterilized if they are naturally sterile or are surgically sterilized.

5 The PPR category also contains the small proportion of teens who use a variety of other female-controlled methods, including emergency contraception, diaphragms, female condoms, foams, jellies/creams, suppositories/inserts, the contraceptive sponge, and natural family planning. Approximately $2 \%$ of teens in the PPR category in the 2011-2013 NSFG were using one of these other methods.

6 Condoms and withdrawal have similar levels of estimated effectiveness (Trussell, 2011). For purposes of simplicity, we therefore collapse condom users and users of withdrawal into a single "condom" category. As is the case for female sterilization, we consider teen women to rely on male sterilization if their partners are surgically sterilized or are naturally sterile. 
Thus, as analysis time passes, some non-contracepting teens will begin to use contraception, and some contracepting teens will discontinue contraceptive use or switch to a different method.

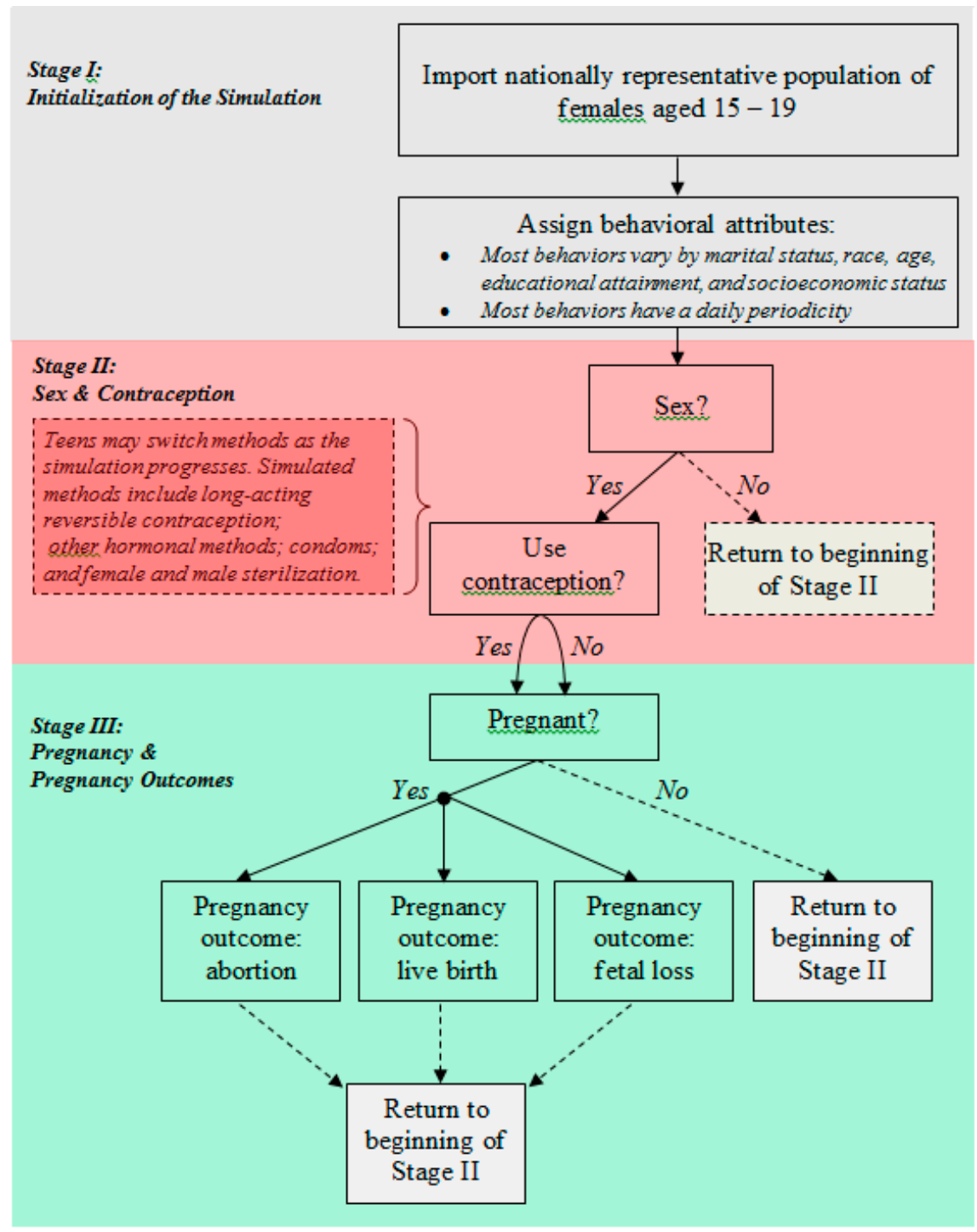

Figure 1. Summary diagram of the Teen FamilyScape Microsimulation Model.

Teen FamilyScape's third and final stage models the occurrence of pregnancy. A teen's risk of pregnancy when she has sex is dependent on her underlying fecundity (i.e., her probability of experiencing a pregnancy from a single act of unprotected intercourse) and the efficacy of any contraception that she is using. Fecundity varies according to a girl's age and the day in her menstrual cycle. Thus, on each new day within the simulation, we update each girl's menstrual calendar and modify her age-adjusted pregnancy probability accordingly. The model's fecundity parameters were developed by synthesizing the results of several fertility studies (see Thomas and Karpilow (2015) for more information on these fertility studies) [11].

We developed Teen FamilyScape's contraceptive efficacy parameters by combining information on the age-dependent fecundities, coital frequencies, and typical-use pregnancy rates of girls in each contraceptive category. We estimated fecundities and coital frequencies using NSFG data, and we produced method-specific pregnancy rates by combining a number of different published estimates. More specifically, for each contraceptive method incorporated into our simulations, we: (a) used data reported in Trussell et al., (1997) to compute a ratio of the pregnancy rate among teen users of 
the method in question to the pregnancy rate among all users of that method; and (b) multiplied this ratio by Trussell's (2011) more recently estimated method-specific pregnancy rate for all women [7,13]. This allowed us to take advantage of Trussell et al.'s teen-specific estimates (which are the most recent of which we are aware) and to update those estimates under the simplifying assumption that there has been no change over time in the proportional difference between the method-specific pregnancy rates experienced by teen contraceptors and all contraceptors. We then calculated weighted averages of these teen-specific, method-specific pregnancy rates for each of the model's contraceptive categories, where the weights reflected the share of teens in a given category who use each of the methods falling into that category. Thus, for example, our final failure rate for the LARC/injectable method category is a weighted average of the pregnancy rates experienced by teenage women who use IUDs, implants, and injectables. Our weights were constructed using age-specific estimates of the distribution of methods used at last sex as reported by Jones et al., [14]. The only exceptions to this rule are male and female sterilization, both of which we always assume to be $100 \%$ effective.

We would also note that, because of sample-size limitations, we do not model separate contraceptive efficacy rates for dual-method and single-method users. Our efficacy parameters instead reflect the average risk of pregnancy across dual-method and single-method users. As such, we assign to each member of the simulation population an efficacy rate that corresponds to the most effective method (if any) that she is using. Thus, for example, we use PPR efficacy rates to model the risk of pregnancy among pill users (whether or not they are also using condoms), and we use condom efficacy rates to model the risk of pregnancy among teen women who rely on condoms and are not using a female-controlled method. We followed the approach outlined in Thomas and Karpilow (2015) to model pregnancy risk among non-contraceptors [11].

Every simulated pregnancy eventually results in a birth, an abortion, or a fetal loss (i.e., a miscarriage). We used data reported in Ventura et al., (2012) to develop the model's pregnancy-outcome parameters [15]. For each pregnancy, we also simulate a gestation period and an interval of post-pregnancy infertility, both of which vary according to the pregnancy's outcome (see Thomas and Karpilow (2015) for more information) [11]. Although an individual may continue to have sex while she is pregnant, she is not at risk of pregnancy until after her interval of post-pregnancy infertility has ended.

Teen FamilyScape can be validated by comparing its outputs to their equivalent real-world benchmarks. Table 1 compares simulated and real-world rates of teenage pregnancy, childbearing, and abortion. For all three outcomes, the model closely approximates the relevant real-world benchmark ${ }^{7}$.

Table 1. Pregnancy and pregnancy outcome rates, 2008.

\begin{tabular}{lcc}
\hline & Teen FS & Ventura et al. \\
\hline Pregnancies per 1000 teens & 70.0 & 69.8 \\
Births per 1000 teens & 40.8 & 40.2 \\
Abortions per 1000 teens & 17.2 & 17.8 \\
\hline
\end{tabular}

\subsection{Description of Simulation Specifications and Results}

We used Teen FamilyScape to model the effects of historical changes in teen women's contraceptive use on the teen pregnancy rate. The first and fourth columns of Table 2 report the distribution of contraceptive use among teenage female respondents in the 2002 and 2011-2013 NSFG samples. As was done in previous research [4], we focused in particular on the method used at last sex among respondents who were sexually active in the three months prior to the survey. Based on these distributions, the share of sexually active teens who failed to use contracgeption at last sex fell by

7 Benchmarks were taken from Ventura et al., (2012). 
4.9 percentage points over the past decade (from $17.1 \%$ to $12.2 \%$ ). This reduction in non-contraception was accompanied by an increase in condom, PPR, and LARC/injectable method use. The condom category experienced the largest percentage-point increase, followed by the PPR category and the LARC/injectable category. Additional analyses of the NSFG (not shown here) found that, between 2002 and 2011-2013, this 4.9 percentage-point increase in contraceptive use was accompanied by a similar reduction of 4.7 percentage points in the number of sexually active teens (those having sex in the past three months), suggesting that both factors were associated with declines in teen pregnancy over this time period.

Table 2. Decomposition of changes in teenage contraceptive use between 2002 and 2011-2013 and simulated effects on the teen pregnancy rate.

\begin{tabular}{|c|c|c|c|c|c|}
\hline $\begin{array}{l}\text { Most Effective Method } \\
\text { Used at Last Sex }\end{array}$ & $\begin{array}{c}\text { (1) } 2002 \\
\text { Distribution }\end{array}$ & $\begin{array}{l}\text { (2) } 2002 \\
\text { Distribution }+ \\
\text { Increased } \\
\text { Condom Use }\end{array}$ & $\begin{array}{c}\text { (3) } 2002 \\
\text { Distribution }+ \\
\text { Increased Condom } \\
\text { and PPR Use }\end{array}$ & $\begin{array}{c}\text { (4) 2011-2013 Distribution: } \\
2002 \text { Distribution + Increased } \\
\text { Condom, PPR and } \\
\text { LARC/Injectable Method Use }\end{array}$ & Total \\
\hline Sterilization & $0.7 \%$ & $0.2 \%$ & $0.0 \%$ & $0.0 \%$ & - \\
\hline LARC/injectable & $9.8 \%$ & $9.8 \%$ & $9.8 \%$ & $10.1 \%$ & - \\
\hline IUD/Implant & $0.6 \%$ & $0.6 \%$ & $0.6 \%$ & $3.3 \%$ & - \\
\hline Injectable & $9.2 \%$ & $9.2 \%$ & $9.2 \%$ & $6.8 \%$ & - \\
\hline Pill, patch, ring (PPR) & $36.0 \%$ & $36.0 \%$ & $37.2 \%$ & $37.2 \%$ & - \\
\hline Condom & $36.4 \%$ & $40.5 \%$ & $40.5 \%$ & $40.5 \%$ & - \\
\hline No method & $17.1 \%$ & $13.5 \%$ & $12.5 \%$ & $12.2 \%$ & - \\
\hline $\begin{array}{l}\text { Simulated reduction in } \\
\text { the number of } \\
\text { pregnancies per } \\
1000 \text { teen women }\end{array}$ & - & -4.7 & -1.7 & -1.8 & -8.1 \\
\hline $\begin{array}{c}\% \text { of the Total } \\
\text { Contraceptive Effect }\end{array}$ & - & $57.6 \%$ & $20.6 \%$ & $21.8 \%$ & $100 \%$ \\
\hline
\end{tabular}

Note: The italic numbers represent a breakdown of the LARC/injectable category.

Our objective was to develop separate estimates of the effects of the increases in teenage women's use of condoms, PPR methods, and LARC/injectable methods between 2002 and 2011-2013 on declines in teen pregnancy rates. We therefore began by re-parameterizing Teen FamilyScape to replicate the 2002 distribution of method use at last sex as reported in column (1) of Table 2 . We then implemented a series of intermediate simulation specifications in which we successively aligned the proportion of teens who use each of these three method types with the corresponding benchmark from the 2011-2013 distribution. For all three simulations, we moved a subset of teens out of the "sterilized" and "no method" categories, which are the two categories that shrank in size during our period of interest. We specified these reductions in sterilization and non-contraception so as to ensure that we were ultimately able to replicate the 2011-2013 distribution of teenage contraceptive use. Table 2 shows that, between 2002 and 2011-2013, the share of teens who were sterilized fell by 0.7 percentage points, while the share of teens who were noncontraceptors fell by 4.9 percentage points. Thus, we ultimately simulated changes in contraceptive use for $5.6 \%(0.7 \%+4.9 \%)$ of teens ${ }^{8}$. After implementing these three specifications, we arrived at the 2011-2013 distribution of teen method use. The change in the teen pregnancy rate from one specification to the next reflects the estimated effect of the corresponding change in contraceptive use between 2002 and 2011-2013.

8 Among the members of this group, $12.5 \%$ originally fell into the "sterilized" category, and $87.5 \%$ originally fell into the "no method" category. When we simulated flows out of these two categories, we therefore always made certain that $12.5 \%$ of the affected teens were originally in the "sterilization" category and that $87.5 \%$ of the affected teens were originally in the "no method" category. In so doing, we ensured that our simulations ultimately reproduced the 2011-2013 distribution of method used at last sex. 
For the first of our three simulations, we started with the 2002 distribution of contraceptive use and then moved a subset of sterilized and non-contracepting teens in the Teen FamilyScape model into the condom use category, which yielded the distribution shown in column (2). In this distribution, the share of teens who were condom users was the same as in the 2011-2013 distribution; the share of teens who were sterilized or non-contraceptors was smaller than in the original 2002 distribution; and the shares of teens who were PPR and LARC/injectable method users were held constant at their 2002 levels. The difference between the simulated teen pregnancy rates produced by the distributions shown in columns (1) and (2) was our estimate of the effect of the increase in condom use between 2002 and 2011-2013, a decrease of almost five pregnancies per 1000 teen women.

For the second simulation, we began with the distribution in column (2) and then moved a subset of the remaining sterilized and non-contracepting teens into the PPR category. This specification yielded the distribution shown in column (3), in which the shares of teens who were condom and PPR users were the same as in the 2011-2013 distribution, while the share of teens who were LARC/injectable method users was again unchanged relative to the 2002 distribution. The difference between the simulated teen pregnancy rates produced by the distributions shown in columns (2) and (3) was therefore our estimate of the effect of the increase in PPR use between 2002 and 2011-2013, a decrease of approximately two pregnancies per 1000 teen women.

Finally, for the third simulation, we began with the distribution in column (3) and then moved a subset of the remaining sterilized and non-contracepting teens into the LARC/injectable method category, which yielded the 2011-2013 distribution of contraceptive use shown in column (4). The difference between the simulated teen pregnancy rates produced by the distributions in columns (3) and (4) was our estimate of the effect of increased LARC/injectable method use, a decrease of approximately two pregnancies per 1000 teen women.

Note that Teen FamilyScape combines LARCs and injectables into a single category encompassing IUDs, implants, and injectables. The efficacy rates for IUDs and implants are higher-and their discontinuation rates are lower-than for injectables. Among teens who used one of these three methods, the share who relied specifically on IUDs or implants rose from less than $10 \%$ in 2002 to nearly one third in 2011-2013. For the simulation described above, we adjusted the LARC/injectable category's efficacy and discontinuation rates in order to account for changes over time in mix of LARC/injectable methods used by teens ${ }^{9}$. We did not make similar adjustments when we simulated increased PPR use because the estimated efficacy and discontinuation rates for the pill, patch, and ring are very similar [7]. We would also note that, aside from adjusting efficacy and discontinuation rates within the LARC/injectable category, we did not model any other changes over time in the probability of contraceptive discontinuation or in the consistency of correctness of method use. Nor did we simulate the effects of changes in coital frequency or in teenagers' demographic characteristics. This is because our specific purpose was to isolate the effects of changes in contraceptive use during a typical act of intercourse, holding all else constant.

Overall, we found that changes in contraceptive use produced a reduction of 8.1 pregnancies per 1000 teens in the teen pregnancy rate, which corresponds to a little less than half of the decline in teen pregnancies during our period of interest (17 pregnancies per 1000 teenage women between 2002 and 2010) $[1]^{10}$. Our results imply that more than half (about 58\%) of this contraceptive effect was driven by increased condom use and that the remainder was attributable in roughly equal measure

9 Because the contraceptive efficacy and switching parameters for Teen FamilyScape's base specification were developed using NSFG data from 2006-2010, and since injectable use was more common among teenaged LARC/injectable users in 2002 than in 2006-2010, we made comparable adjustments to the model's LARC/injectable efficacy and switching rates when we parameterized the model to reproduce the 2002 distribution of contraceptive use.

10 The most recent pregnancy estimates are from 2010. Based on historical trends in the teen pregnancy rate and the continued decline in the teen birth rate between 2010 and 2012, we anticipate that the teen pregnancy rate might have been even lower by 2012 in which case the magnitude of the contraceptive effect would be reduced as we study contraceptive changes from 2002 to 2011-2013. 
to increased PPR and LARC/injectable method use ${ }^{11}$. Other simulation results (not shown here) indicated that about $30 \%$ of the "LARC/injectable method effect" was attributable to the growth in the number of teens who used an IUD, an implant, or an injectable. The remaining $70 \%$ of the effect was attributable to the fact that, among teens who used these three methods, there has been an increase in the share who rely more specifically on IUDs and implants rather than injectables.

\subsection{How Important Was the Decline in Non-Contraception?}

During our period of study, increases in the use of effective methods were accompanied by a substantial reduction in the number of non-contracepting teens. To understand the relative importance of the decline in non-contraception as compared to the increase in the use of effective methods, we performed a final counterfactual simulation in which we modeled a change in contraceptive behavior for the same number of teens as in our main specifications (approximately $5 \%$ of sexually active teens), but we assumed that the number of non-contraceptors remained constant. We instead achieved the desired change in contraceptive use by moving a subset of condom users onto more effective methods.

The top panel of Table 3 shows our simulated counterfactual distribution of method use. We also restate the 2002 distribution that is reported in Table 2. As was the case in our earlier simulations, we once again move $0.7 \%$ of teens out of the sterilization category. However, whereas our main specifications assumed a 4.9 percentage-point reduction in the share of teens who were non-contraceptors, we assumed instead for this simulation that there was a 4.9 percentage-point reduction in the share of teens who used condoms, as well as a 0.7 percentage point decline in sterilization. All of these "former condom users" were moved into the PPR and LARC/injectable method categories. We assumed that the ratio of new LARC/injectable method to PPR users was identical to the equivalent ratio as measured in our real-world 2011-2013 distribution (see Table 2, column 4). Under this assumption, the sizes of the LARC/injectable method and PPR categories increased by 1.2 and 4.4 percentage points, respectively. As was the case for our earlier simulations, we also modeled increases in contraceptive efficacy and reductions in discontinuation rates among LARC/injectable method users in order to account for changes over time in the mix of LARC/injectable methods that are used by teens.

Table 3. Counterfactual simulation of changes in teenage contraceptive use.

\begin{tabular}{ccc}
\hline Most Effective Method Used at Last Sex & (1) $\mathbf{2 0 0 2}$ Distribution & $\begin{array}{c}\text { (2) Counterfactual } \\
\text { Distribution }\end{array}$ \\
\hline Sterilization & $0.7 \%$ & $0.0 \%$ \\
LARC/injectable method & $9.8 \%$ & $11.0 \%$ \\
Pill, patch, ring (PPR) & $36.0 \%$ & $40.4 \%$ \\
Condom & $36.4 \%$ & $31.5 \%$ \\
No method & $17.1 \%$ & $17.1 \%$ \\
Simulated Reduction in the Number of Pregnancies per $\mathbf{1 0 0 0}$ Teenaged Women & $-\mathbf{3 . 0}$ \\
\hline
\end{tabular}

Note: Contraceptive distributions are based on the authors' analysis of data from the 2002 and 2011-2013 National Surveys of Family Growth. Estimated contraceptive effect is based on the results produced by the Teen FamilyScape Microsimulation model.

We found that, when we moved from the 2002 distribution of method use to the counterfactual distribution shown above, the teen pregnancy rate was reduced by 3.0 pregnancies per 1000 teenage women. This effect was only about $37 \%$ as large as the effect that was produced when we modeled

11 Because these three simulations are additive in nature, their order has no effect on our results. Our estimates of the effects of increased condom, PPR, and LARC/injectable method use would have been the same if (for example) we had simulated an increase in LARC/injectable method use, then in condom use, and then in PPR use. 
the change from the 2002 distribution to the actual 2011-2013 distribution (an overall decline of 8.1 pregnancies per 1000 women). In other words, moving non-contracepting teens onto condoms had a larger impact on the teen pregnancy rate than moving teen condom users onto PPR and LARC/injectable methods. Thus, our analyses indicate that the decline in teen pregnancy rates between 2002 and 2011-2013 was driven primarily by reductions in non-contraception, rather than by reductions in the use of less effective methods.

\section{Discussion}

There have been dramatic declines in teen pregnancy rates in the past decade, and previous research suggests that improvements in teen contraceptive use have played a major role in these declines [3-6]. A better understanding of the implications of changes in contraceptive behavior for historical declines can inform policymakers and practitioners as to the most effective strategies for sustaining the reduction in the rate of teen pregnancy, which remains high compared to other industrialized countries [16]. Our analyses indicate that approximately half of the decline in teen pregnancies since 2002 was due to changes in contraceptive method use ${ }^{12}$. This estimate fits within the range of other studies, which found that changes in contraceptive use accounted for between $47 \%$ and $86 \%$ of declines in teen pregnancy and childbearing [4,5]. Differences between our findings and those of other studies may be due, in part, to the more recent time period of our study (previous work highlighted trends in sexual activity and contraceptive use since the early 1990s and did not extend past the early 2000s). Our estimate of the contraceptive effect may, in fact, be a lower bound because we focus only on the effect of changes in methods used at a typical act of intercourse and do not model changes in other dimensions of contraceptive behavior. If changes in the mix of contraceptive methods were accompanied by, for instance, reductions in discontinuation rates, improvements in the consistency or correctness of use, or changes in dual method use ${ }^{13}$, we might have found that changes in contraceptive behavior had an even more substantial impact on trends in teen pregnancy. For instance, Santelli et al. 2007 modeled a reduction in nonuse as well as increased use of more effective methods and of multiple methods simultaneously and found a larger contraceptive effect. Moreover, recent research has documented an increase in dual method use among teens in recent years [6].

Our work extends previous research by examining how changes in the mix of contraceptive methods are linked to declines in teen pregnancy. Previous research has found that the percentage of sexually active teens not using contraception declined from 1998 to 2006-2010 [17], and we found that these declines have continued into 2011-2013. Declines in nonuse have been accompanied by increases in condom use and pill, patch or ring (PPR) use, and slight increases in LARC/injectable methods-all of which contributed to declines in teen pregnancy rates in the past decade.

Condoms were the most frequently used contraceptive method among sexually active teens, with more than $40 \%$ reporting condoms as their most effective method in 2011-2013. The increase in condom use since 2002 accounts for more than half of the contraceptive effect on declines in teen pregnancy rates in our models. Increases in the use of PPR methods and LARC/injectable methods account for the remaining contraceptive effect in equal measure. Our analyses highlight slight increases in teen use of PPR contraceptive methods during the time period of study, with $37 \%$ of teen women relying on these methods in 2011-2013. Our analyses also indicate an overall

12 We did not explicitly model changes in sexual behavior or attempt to explain the remaining portion of the historic decline in teen pregnancy. However, we did find an almost five percent point reduction in the number of sexually active tens (those having sex in the past three months), suggesting that both increased contraceptive use and decreased sexual activity were associated with declines in teen pregnancy over this time period.

13 Teen FamilyScape accounts for dual method use in that the failure rates for the PPR and LARC/injectable method groups are weighted averages of the failure rates experienced by dual-method and single-method users. The model, however, does not account for changes in dual method use over time. Thus, we implicitly assume that dual method use patterns remained constant. 
slight increase in the LARC/injectable methods (injectables, IUDs and implants). However, this overall slight increase in the combination of injectables and LARCs masks a decline in the use of injectable methods that was accompanied by a larger increase in LARCs (IUDs and implants). In fact, while LARC use is still very low among teens (about 3\% used an IUD or implant in 2011-2013), it has increased by a factor of more than five since 2002 (see Table 2).

Additional simulation analyses highlighted that the contraceptive effect was driven primarily by the drop in the percentage of teens using no method, rather than by the increase in the use of more effective methods. When we modeled only changes in the method mix among contraceptors, leaving the percentage of teens using no method at 2002 levels, we found that the magnitude of the contraceptive effect dropped by two-thirds from a decline of 8.1 pregnancies per 1000 teen women to a decline of three pregnancies per 1000 teen women. Women who do not use contraception have a very high rate of pregnancy: an average of $85 \%$ of sexually active women experience pregnancy over the course of a year. Thus, we find that take-up of even less effective methods among teens can result in a dramatic reduction in the teen pregnancy rate [7].

Our findings suggest the need for a two-pronged approach to continue declines in teen pregnancy among sexually active teens into the future. First, policies and programs should continue to target sexually active youth who do not use contraception. Second, teen pregnancy rates could decline further if policymakers can increase the effectiveness of method use among existing contraceptors-for example, by providing evidence-based contraceptive counseling, affordable services and same-day prescriptions/insertions [18-20].

Despite recent declines, more than one in ten sexually active teens in 2011-2013 (12\%) did not use any method of contraception at last sex, highlighting the need for targeted efforts to improve contraceptive use among this population. Reasons for non-use among teens include concern about side effects (for hormonal and LARC methods), misunderstanding of the risk of pregnancy associated with unprotected intercourse, being "in the moment" and not wanting to break the mood, and partner resistance to the use of contraception [21-23]. Many currently non-contracepting teens have used contraceptive methods in the past, as is evidenced by the high rates of sexually active teens who have ever used condoms (97\%) and the pill (54\%) [24]. While method switching can lead to gaps in contraceptive coverage and increase the risk of unplanned pregnancy [25], research suggests that providers can help to prevent gaps in contraceptive coverage by providing teens with multiple contraceptive options and helping them switch to another effective method if they are unhappy with their current method [25]. Additionally, teens who do not use contraception often engage in other risky behaviors such as alcohol and drug use and are more likely to disconnected from school and family, highlighting the need for multiple and combined intervention efforts [26].

A review of effective teen pregnancy prevention studies has documented several programs that have increased condom use among teens who were not using contraception, including very short STD prevention programs and longer, more intensive youth development programs [27]. However, typical use failure rates for condoms are relatively high (at 18\%), and may be even higher for teens [28,29], and many researchers have found that condom use declines as relationships become longer or more serious [30,31], suggesting that transitioning teens to more effective hormonal or long acting-methods of contraception can help teens avoid pregnancy.

Among more effective contraceptive methods, PPR methods remain popular among teens. These methods provide high levels of protection when used perfectly $(0.3 \%$ failure rate), although typical use pregnancy rates are higher (9\%) [7-9], and many women ultimately switch off of these methods [32-34], highlighting the importance of consistent and sustained method use. In the LARC/injectable method category, our study found a decline in injectable method use since the early 2000s. Qualitative research and interviews with providers indicate that clinics are more likely to prescribe and deliver injectables on-site versus other longer-acting methods. However, side effects and difficulty with attending regular appointments have led to high discontinuation rates among injectable users [23]. Although not currently as popular, LARCs (implants and IUDs) are 
recommended as a first-line method for teens, as they combine effectiveness and consistency and have very low typical use failure rates (less than 1\%) [35]. Additionally, despite higher upfront costs, these methods are cost-effective for women who do not intend to get pregnant for several years as their use does not require regular health care visits or prescription refills [36]. Evidence suggests that the low level of LARC use in the United States is attributable in part to a lack of information regarding their benefits, misinformation about their likely side effects, and their high upfront costs [11,12]. However, evaluations of recent LARC-based interventions have found that young women are more likely to choose LARC methods when they are well-informed as to their benefits and potential side effects, same-day insertions are available, and cost barriers are removed $[19,20]$.

\section{Limitations}

Our work has some limitations, mainly related to the Teen FamilyScape model. The model is an accurate and powerful tool for answering our research question-how changes in the use of specific contraceptive methods were associated with the historical decline in teen pregnancy. As noted in the Discussion, however, our estimate of the overall contraceptive effect does not incorporate changes over time in contraceptive behaviors such as dual method use and consistency and correctness of method use. The model also combines LARCs and injectable methods, which is not ideal given the differences in failure and discontinuation rates for these method types. However, we account for this by adjusting failure and discontinuation rates accordingly and are able to capture the relative increase in IUD users over time. Despite these limitations, our model allows us to extend previous research by examining how trends in contraceptive method use have contributed to recent declines in teen pregnancy in the U.S.

\section{Conclusions}

Our work contributes uniquely to the literature on the declining U.S. teen pregnancy rate by parsing out the effect of changes in contraceptive use. The study's findings highlight the importance of targeting pregnancy prevention efforts towards teens who do not use contraception as well as efforts to improve the effectiveness of teens' chosen birth control methods.

Acknowledgments: This research was supported by funding from the Brookings Institution and from Child Trends.

Author Contributions: Quentin Karpilow and Adam Thomas conceived of the modeling process, Quentin Karpilow completed the modeling, constructed the tables, and wrote the the first draft of methods and results. Jennifer Manlove helped conceptualize the analyses, wrote the introduction, literature review and discussion and aided in the construction of the remainder of the paper, as well as the revisions. Adam Thomas and Kate Welti added to the introduction, literature review and discussion sections, edited all sections, and aided in the completion of the manuscript and the interpretation of the findings. All authors have read and approved the final manuscript.

Conflicts of Interest: The authors declare no conflict of interest.

\section{A. Appendix}

The National Survey of Family Growth (NSFG) 2006-10 is a survey of women and men aged 15-44 conducted between 2006-2010. The NSFG contains a sample of women and men from all states in the United States. Resulting statistics are nationally representative when sampling weights are applied. The 2006-2010 NSFG includes 2284 teenaged women ages 15-19. We used information from the NSFG to estimate Teen FamilyScape's parameters related to the probability that a teen woman would have sex in a given month and her monthly coital frequency. We also used the NSFG to assign each woman to an initial contraceptive method and to develop our estimates of contraceptive efficacy. We then used the 2002 and 2011-2013 versions of the survey to calculate the distribution of women using each type of contraceptive method during these two time periods. The table below summarizes the relevant measures: 


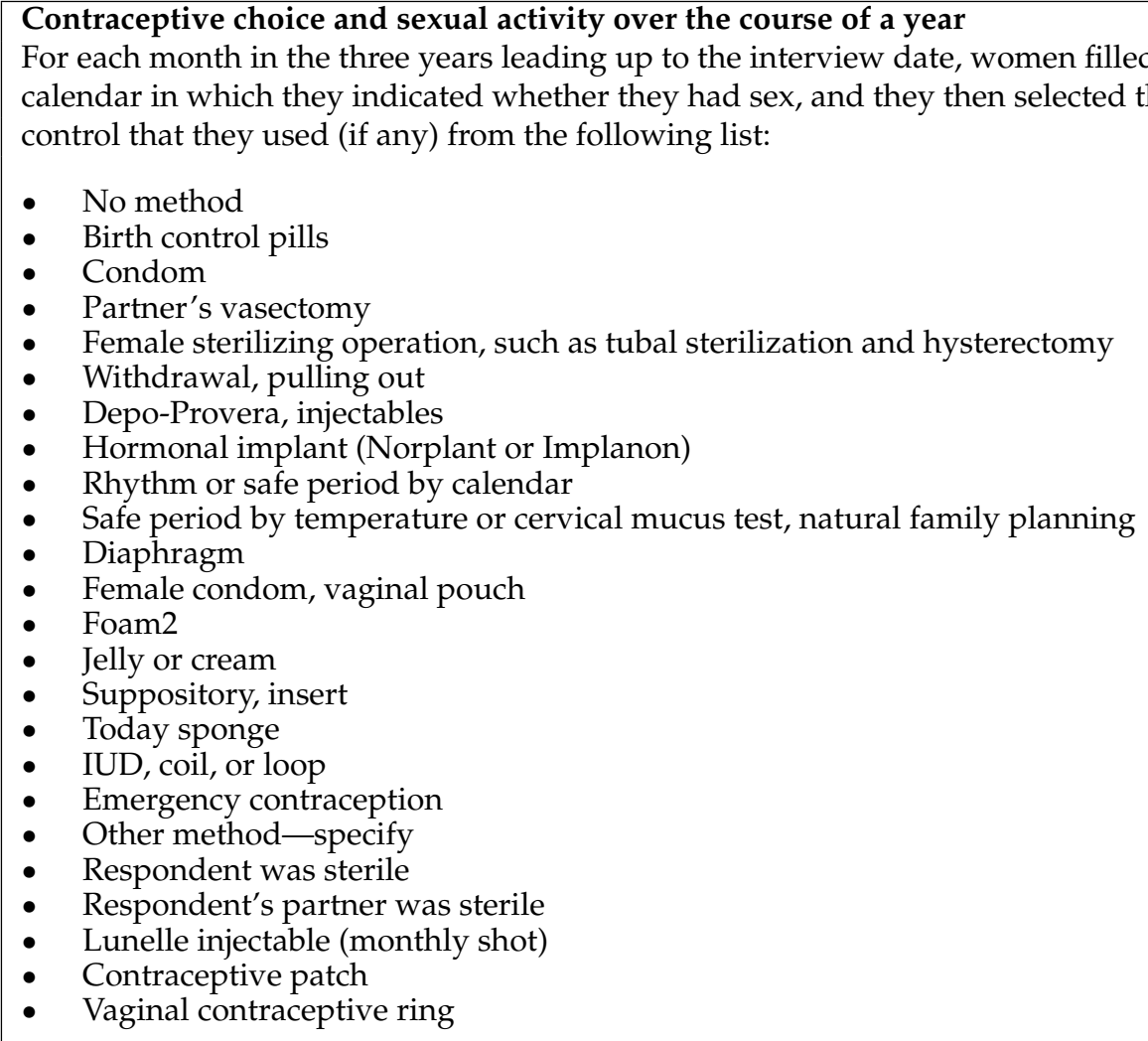

The number of sexually active months in a year is calculated using this calendar and the woman's selected method of birth control is drawn from the first month of the past year in which the woman was sexually active and not pregnant.

\section{References}

1. Kost, K.; Henshaw, S. U.S. Teenage Pregnancies, Births and Abortions, 2010: National Trends by Age, Race and Ethnicity; Guttmacher Institute: New York, NY, USA, 2014.

2. Mohn, J.K.; Tingle, L.R.; Finger, R. An analysis of the causes of the decline in non-marital birth and pregnancy rates for teens from 1991 to 1995. Adolesc. Fam. Health 2003, 3, 39-47.

3. Santelli, J.S.; Abma, J.; Ventura, S.J.; Lindberg, L.; Lyss, S.; Hamilton, B.E. Can changes in sexual behaviors among high school students explain the decline in teen pregnancy rates in the 1990s. J. Adolesc. Health 2004, 35, 80-90. [CrossRef]

4. Santelli, J.S.; Lindberg, L.D.; Finer, L.B.; Singh, S. Explaining recent declines in adolescent pregnancy in the United States: The contribution of abstinence and improved contraceptive use. Am. J. Public Health 2007, 97, 150-156. [CrossRef] [PubMed]

5. Kearney, M.S.; Levine, P.B. Explaining Recent Trends in the U.S. Teen Birth Rate; University of Michigan, National Poverty Center: Ann Arbor, MI, USA, 2012.

6. Boonstra, H.D. What is behind the declines in teen pregnancy rates? Guttmacher Policy Rev. 2014, 17, $15-21$.

7. Trussell, J. Contraceptive efficacy. In Contraceptive Technology; Hatcher, R., Trussell, J., Nelson, A., Cates, W., Jr., Stewart, F., Eds.; Arden Media: New York, NY, USA, 2011; Volume 20.

8. Kost, K.; Singh, S.; Vaughan, B.; Trussell, J.; Bankole, A. Estimates of contraceptive failure from the 2002 National Survey of Family Growth. Contraception 2008, 77, 10-21. [CrossRef] [PubMed]

9. Woods, J.L.; Shew, M.L.; Tu, W.; Ofner, S.; Ott, M.A.; Fortenberry, J.D. Patterns of oral contraceptive pill-taking and condom use among adolescent contraceptive pill users. J. Adolesc. Health 2006, 39, 381-387. [CrossRef] [PubMed]

10. Winner, B.; Peipert, J.F.; Zhao, Q.; Buckel, C.; Madden, T.; Allsworth, J.E. Effectiveness of long-acting reversible contraception. New Engl. J. Med. 2012, 366, 1998-2007. [CrossRef] [PubMed] 
11. Thomas, A.; Karpilow, Q. Familyscape 3.0: Architectural Overview; Brookings Institution: Washington, DC, USA, 2015.

12. National Survey of Family Growth. 2011-2013 National Survey of Family Growth, User's Guide; U.S. Department of Health and Human Services: Hyattsville, MD, USA, 2013.

13. Trussell, J.; Koenig, J.; Stewart, F.; Darroch, J. Medical care cost savings from adolescent contraceptive use. Fam. Plan. Perspect. 1997, 29, 248-255. [CrossRef]

14. Jones, J.; Mosher, W.; Daniels, K. Current Contraceptive Use in the United States, 2006-2010, and Changes in Patterns of Use Since 1995; National Center for Health Statistics: Hyattsville, MD, USA, 2012.

15. Ventura, S.J.; Curtin, S.C.; Abma, J.C.; Henshaw, S.K. Estimated Pregnancy Rates and Rates of Pregnancy Outcomes for the United States, 1990-2008; National Center for Health Statistics: Hyattsville, MD, USA, 2012.

16. Sedgh, G.; Finer, L.B.; Bankole, A.; Eilers, M.A.; Singh, S. Adolescent pregnancy, birth, and abortion rates across countries: Levels and recent trends. J. Adolesc. Health 2015, 56, 223-230. [CrossRef] [PubMed]

17. Martinez, G.; Copen, C.E.; Abma, J.C. Teenagers in the United States: Sexual Activity, Contraceptive Use, and Childbearing, 2006-2010 National Survey of Family Growth; National Center for Health Statistics: Washington, DC, USA, 2011.

18. McNicholas, C.; Madden, T.; Secura, G.M.; Peipert, J.F. The contraceptive choice project round up: What we did and what we learned. Clin. Obstet. Gynecol. 2014, 57, 635-643. [CrossRef] [PubMed]

19. Secura, G.M.; Madden, T.; McNicholas, C.; Mullersman, J.L.; Buckel, C.M.; Zhao, Q.; Peipert, J.F. Provision of no-cost, long-acting contraception and teenage pregnancy. New Engl. J. Med. 2014, 371, 1316-1323. [CrossRef] [PubMed]

20. Harper, C.C.; Rocca, C.H.; Thompson, K.; Morfesis, J.; Goodman, S.; Darney, P.D.; Westhoff, C.L.; Speidel, J.J. Reductions in pregnancy rates in the U.S.A. with long-acting reversible contraception: A cluster randomised trial. Lancet 2015, 386, 562-568.

21. Centers for Disease Control and Prevention. Pregnancy Contraceptive Use among Teens with Unintended Pregnancies Resulting in Live Births: Pregnancy Risk Assessment Monitoring System (PRAMS), 2004-2008; U.S. Department of Health and Human Serivces, Centers for Disease Control and Prevention: Atlanta, GA, USA, 2012; pp. 25-29.

22. Brown, S.; Guthrie, K. Why don't teenagers use contraception? A qualitative interview study. Eur. J. Contracept. Reprod. Health Care 2010, 15, 197-204. [CrossRef] [PubMed]

23. Mosher, W.D.; Jones, J. Use of Contraception in the United States: 1982-2008; National Center for Health Statistics: Hyattsville, MD, USA, 2010.

24. Martinez, G.M.; Abma, J.C. Sexual Activity, Contraceptive Use, and Childbearing of Teenagers Aged 15-19 in the United States; National Center for Health Statistics: Hyattsville, MD, USA, 2015.

25. Jaccard, J. Counseling adolescents about contraception: Towards the development of an evidence-based protocol for contraceptive counselors. J. Adolesc. Health 2012, 52, s6-s13. [CrossRef] [PubMed]

26. Kirby, D. Effective approaches to reducing adolescent unprotected sex. J. Sex Res. 2002, 39, 51-57. [CrossRef] [PubMed]

27. Goesling, B.; Colman, S.; Trenholm, C.; Terzian, M.; Moore, K.A. Programs to reduce teen pregnancy, sexually transmitted infections, and associated sexual risk behaviors: A systematic review. J. Adolesc. Health 2014, 54, 499-507. [CrossRef] [PubMed]

28. Trussell, J. Contraceptive failure in the United States. Contraception 2011, 83, 397-404. [CrossRef] [PubMed]

29. Fu, H.; Darroch, J.E.; Haas, T.; Ranjit, N. Contraceptive failure rates: New estimates from the 1995 National Survey of Family Growth. Fam. Plan. Perspect. 1999, 31, 56-63. [CrossRef]

30. Manlove, J.; Welti, K.; Barry, M.; Peterson, K.; Schelar, E.; Wildsmith, E. Relationship characteristics and contraceptive use among young adults. Perspect. Sex. Reprod. Health 2011, 43, 119-128. [CrossRef] [PubMed]

31. Ku, L.; Sonenstein, F.L.; Pleck, J.H. The dynamics of young men's condom use during and across relationships. Fam. Plan. Perspect. 1994, 26, 246-251. [CrossRef]

32. Raine, T.R.; Foster-Rosales, A.; Upadhyay, U.D.; Boyer, C.B.; Brown, B.A.; Sokoloff, A.; Harper, C.C. One-year contraceptive continuation and pregnancy in adolescent girls and women initiating hormonal contraceptives. Obstet. Gynecol. 2011, 117, 363-371. [CrossRef] [PubMed]

33. Littlejohn, K.E. Hormonal contraceptive use and discontinuation because of dissatisfaction: Differences by race and education. Demography 2012, 49, 1433-1452. [CrossRef] [PubMed] 
34. Vaughan, B.; Trussell, J.; Kost, K.; Singh, S.; Jones, R. Discontinuation and resumption of contraceptive use: Results from the 2002 National Survey of Family Growth. Contraception 2008, 78, 271-283. [CrossRef] [PubMed]

35. The American College of Obstetricians and Gynecologists. Adolescents and Long-Acting Reversible Contraception: Implants and Intrauterine Devices; American College of Obstetricians and Gynecologists, Women's Health Care Physicians: Washington, DC, USA, 2012.

36. Trussell, J. Update on and correction to the cost-effectiveness of contraceptives in the United States. Contraception 2012, 85. [CrossRef]

(c) 2015 by the authors; licensee MDPI, Basel, Switzerland. This article is an open access article distributed under the terms and conditions of the Creative Commons by Attribution (CC-BY) license (http:/ / creativecommons.org/licenses/by/4.0/). 